\title{
A jump-diffusion model for pricing electricity under price-cap regulation
}

\author{
M. Kegnenlezom ${ }^{1}$ ·P. Takam Soh ${ }^{1}$ - M. L. D. Mbele Bidima ${ }^{1}$ - Y. Emvudu Wono ${ }^{1}$
}

Received: 26 June 2019 / Accepted: 9 October 2019 / Published online: 19 October 2019

(c) The Author(s) 2019

\begin{abstract}
In this paper, we derive a new jump-diffusion model for electricity spot price from the "Price-Cap" principle. Next, we show that the model has a non-classical mean-reverting linear drift. Moreover, using this model, we compute a new exact formula for the price of forward contract under an equivalent martingale measure and we compare it to Cartea et al. (Appl Math Finance 12(4):313-335, 2005) formula.
\end{abstract}

Keywords Model $\cdot$ Electricity market $\cdot$ Price-cap regulation $\cdot$ Spot price $\cdot$ Forward price

\section{Introduction}

According to [26], no economical development is possible without the availability of energy. Accepting this reality, several governments consider electricity as one of their main priorities. For example in France, electricity is recognized by the law as a basic necessity. Its price is determined by regulated tariffs made by the government [4]. Several changes have been operated in electricity sector. Regulation and deregulation are the main mechanisms which caused these changes observed in electricity sector. The aims were to create a competitive economical environment in which the producers, the investors and the large part of consumers would get their satisfaction.

The introduction of deregulation induced many consequences. One main consequence was the high variation in price which encouraged the development of a new breed of financial products in electricity markets. These new products may help cover both physical and financial risks on the

\footnotetext{
M. Kegnenlezom

kegnenlezom@gmail.com

P. Takam Soh

patricetakam@gmail.com

M. L. D. Mbele Bidima

mbelebidima@gmail.com

Y. Emvudu Wono

yemvudu@yahoo.fr

1 University of Yaounde 1, Yaounde, Cameroon
}

new market. Therefore, there has been an important research effort devoted to electricity price modeling for derivative pricing. Due to the non-storable nature of electricity, the challenge of the researchers was the development of a completely satisfying methodology that would help to obtain realistic and robust models. Two standard approaches have often been used to handle this problem in the literature. The first consists in modeling directly the forward curve dynamics and deduces the spot price $[2,5]$. The second approach starts from a spot price model to derive future prices as the expectation of the spot price under a risk-neutral probability. Relevant contributions have been made by $[8,20]$ in pricing energy derivatives and electricity. They took into account seasonality and mean reversion. However, their model did not take into account the huge and non-negligible observed spikes in the market. Further [3,6] were among the first to consider price spikes using jump-diffusion models. Similar works were done in $[10,14,15,24,25,30]$. Regular increase in electricity prices and crises observed in the unregulated market are a point of focus in the media and raised the question of regulation. Moreover, direct link between the price of electricity and the national strategy of poverty reduction motivated governments to limit electricity prices, so that it can be more accessible. This leads to the reintroduction of price regulation by most governments. For instance, [19] was the first to propose the price-cap regulation to British government. Several works were done to study effect and impact of regulation in electricity network and the wholesale electricity market $[12,16]$. 
The main contribution of this paper is, using the rate of increase given by the price-cap, to construct a spot price model in regulated electricity market. In addition to well-known specific features of electricity such as mean-reverting and spikes, our proposed model captures important characteristics of price-cap regulation: inflation rate and efficiency rate. Furthermore, we compute explicitly the forward price in this electricity model.

The rest of this paper is structured as follows: In section two, we present a review of some recent electricity price models. Section three deals with the formulation of our model. Moreover, in section three, we discuss the mean-reversion feature of our model and further compare it with [3] model. In section five, we present some numerical simulations of our model to illustrate our theoretical results and support our discussion.

\section{Review of some recent electricity models}

Several models on electricity price dynamics have been proposed in the literature, among which the jump-diffusion model. Merton [23] was among the first to work on this class of models. His first model was developed to describe the dynamic return on equity. This model was progressively extended by $[3,8,20,24]$.

Schwartz [8] considered spot prices as a stochastic process with two components represented by

$P_{t}=f(t)+X_{t} ; t \in[0, \infty)$

where $f$ is a deterministic differentiable function and $X_{t}$ is the stochastic component satisfying

$\mathrm{d} X_{t}=-\alpha X_{t} \mathrm{~d} t+\sigma \mathrm{d} W_{t}$,

with $\alpha>0$ representing the speed of mean reversion, $X(0)=x_{0}$ being the initial condition and $W$ being a standard Brownian motion. Applying Itô's formula on (2), they obtained the following dynamic for spot price

$\mathrm{d} P_{t}=\alpha\left(a(t)-P_{t}\right) \mathrm{d} t+\sigma \mathrm{d} W_{t}$,

where

$a(t)=\frac{1}{\alpha} f^{\prime}(t)+f(t)$.

In the same paper, they also considered log of spot prices, i.e., $\ln P_{t}=f(t)+Y_{t}$ where $Y_{t}$ follows process (2). In this case, they obtained

$\mathrm{d} P_{t}=\alpha\left(b(t)-\ln P_{t}\right) P_{t} \mathrm{~d} t+\sigma \mathrm{d} W_{t}$,

where

$b(t)=\frac{1}{\alpha}\left(\frac{\sigma^{2}}{2}+f^{\prime}(t)\right)+f(t)$.
This model captures mean-reverting feature which is one of the main characteristics of electricity, but the model has failed to take into account the spikes which can occur in electricity markets. Cartea and Figueroa [3] in the same context of deregulation markets extended (4) by adding a jump term and obtained a mean-reverting and jump-diffusion model. They supposed that the spot price process is in the form $\ln S_{t}=g(t)+Y_{t}$, where $g$ is a seasonal deterministic function and that $Y_{t}$ follows a stochastic process given by

$\mathrm{d} Y_{t}=-\alpha Y_{t} \mathrm{~d} t+\sigma \mathrm{d} W_{t}+\ln J \mathrm{~d} q_{t}$.

Using Itô's formula and equation (5), [3] obtained the following model:

$\mathrm{d} S_{t}=\alpha\left(\rho(t)-\ln S_{t}\right) S_{t} \mathrm{~d} t+\sigma(t) S_{t} \mathrm{~d} W_{t}+S_{t}(J-1) \mathrm{d} q_{t}$,

where

$\rho(t)=\frac{1}{\alpha}\left(g^{\prime}(t)+\frac{1}{2} \sigma^{2}(t)\right)+g(t)$,

$J$ is the proportional size of jump and $q_{t}$ is the Poisson process. Hence, on contrary to $[3,8]$ in their model considered the non-constant volatility, jump and deterministic part of spot price as a seasonal function of time.

\section{Model derivation and the main result}

Our model is partly inspired from the electricity price-cap regulation proposed by Littlechild [19] that we recall as follow.

\section{Price-cap market regulation}

The price-cap regulation is an economical principle which aims to establish an incentive scheme for the regulated market. A key objective is to enable companies to maximize the well-being while seeking to maximize their own interests, see [1]. Its principle is to cap the market price. The main components of the price cap include the efficiency factor $(G)$, for transferring the gains to consumers through the reduction of costs; the inflation rate $(I)$, which drives the price changes; the exogenous factors such as customer portion of earnings' sharing $(E)$, service quality penalties $(H)$ and flow-through and uncontrollable costs, if any $(F)$. ENMAX [11] proposed price-cap formula:

$\frac{P_{i}-P_{i-1}}{P_{i-1}}=I_{i}-G_{i}+\left(\frac{-E_{i}-H S_{i}+F_{i}}{P_{i-1}}\right)$,

where $P_{i}$ represents the current year's price and $P_{i-1}$ preceding year price. Later, we would be inspired by the economic formula (7) to model the drift of the model. 


\section{Spot price modeling procedure}

The daily (resp. weekly and monthly) change in price is the difference between today's price (resp. this week's price and this month's price) and yesterday's price (resp. last week's price and last month's price). In general, one denotes a change over a given time period $\mathrm{d} t$ by $\mathrm{d} S_{t}$. For a daily change, we therefore have $\mathrm{d} t=\frac{1}{365}, \mathrm{~d} t=\frac{1}{52}$ for weekly change and $\mathrm{d} t=\frac{1}{12}$ for a monthly change. The change in price $\mathrm{d} S_{t}$ over a given time period $\mathrm{d} t$ is the sum of two components: the "drift" term and the stochastic (or "random") term, that is,

$\mathrm{d} S=$ drift term + stochastic term.

The drift term represents the portion of the movement in the spot price $S$, which we expect to see with certainty. This term is proportional to the time period over which the change in the price is measured. That is,

drift $\propto \mathrm{d} t$.

The stochastic term represents the portion of the change that is random and cannot be predicted. This term is proportional to the increment $\mathrm{d} W_{t}$ of standard Brownian motion, which is normally distributed with mean zero and variance $\mathrm{d} t$ (see [28, pp. 377-380]. That is,

stochastic term $\propto \mathrm{d} W_{t}, \quad \mathrm{~d} W_{t} \sim \mathscr{N}(0, \mathrm{~d} t)$.

\section{The main result}

Before stating the following theorem, let us recall that a càdlàg stochastic process is the right continuous with leftlimit stochastic process.

Theorem 1 Suppose that the spot price $S_{t}$ is a càdlàg process in a complete filtered probability space $\left(\Omega, \mathscr{F},\left(\mathscr{F}_{t}\right)_{0 \leq t \leq T}, \mathbb{P}\right)$ where $\left(\mathscr{F}_{t}\right)_{t}$ is a natural filtration of $S_{t}$. Assume the following conditions:

(i) for a small time interval $\Delta t$, the change in the electricity price is proportional to $\Delta t$,

(ii) the inflation rate I always differs from the efficiency factor $G$,

(iii) the stock prices jumps from the previous value $S_{t^{-}}$to a next value $J S_{t^{-}}$where $J$ is the proportional size of the random jump assumed log-normally distributed, i.e., $\ln J \sim \mathscr{N}\left(m_{J}, \sigma_{J}^{2}\right)$ with $\mathbb{E}[J]=1$,

(iv) the change before and after the jumps is driven by increments $\mathrm{d} q_{t}$ of a Poisson process $q_{t}$ defined by

$$
\mathrm{d} q_{t}= \begin{cases}1, & \text { with probability } \ell \mathrm{d} t \\ 0, & \text { with probability } 1-\ell \mathrm{d} t\end{cases}
$$

where $\ell$ is the intensity or frequency of the process.

Then, the price-cap principle (7) yields the stochastic differential equation (SDE) below

$\mathrm{d} S_{t}=-\alpha(t)\left(\gamma(t)-S_{t}\right) \mathrm{d} t+\sigma(t) S_{t} \mathrm{~d} W_{t}+(J-1) S_{t} \mathrm{~d} q_{t}$,

where $W_{t}$ is the standard Brownian motion and the coefficients involved are deterministic functions of time denoted as such: $\sigma(t)$ is the volatility, $\beta(t):=E(t)+H(t)-F(t)$ defines the exogenous factors, $\alpha(t):=I(t)-G(t)$, and $\gamma(t):=\beta(t) / \alpha(t)$.

Proof For a small time interval $\Delta t$, the change in the electricity price is proportional to $\Delta t$ and the expected change is by (7) therefore we have

$\Delta S=\left[S_{t}(I(t)-G(t))-E(t)-H(t)+F(t)\right] \Delta t$,

where $\Delta S_{t}=S_{t+\Delta t}-S_{t}$. For $\Delta t \rightarrow 0$, we obtain

$\mathrm{d} S_{t}=\left[S_{t}(I(t)-G(t))-E(t)-H(t)+F(t)\right] \mathrm{d} t$.

To take into account market volatility in the model, the stochastic, or random, contribution to the change in the spot price is represented by $\sigma(t) S_{t} \mathrm{~d} W_{t}$ (see [28, pp. 103-104]). Hence, we obtain the following SDE

$\mathrm{d} S_{t}=\left[S_{t}(I(t)-G(t))-\beta(t)\right] \mathrm{d} t+\sigma(t) S_{t} \mathrm{~d} W_{t}$.

Next, to capture the market shocks we add the jump term in (11) using [3] idea as follows. We suppose that the stock prices jump from the previous value $S_{t^{-}}$to a next value $J S_{t^{-}}$ where $J$ is the proportional size of the random jump assumed log-normally distributed such that $\mathbb{E}(J)=1$ this assumption is motivated by the fact that under regulation we want that the risk of the market shocks fluctuates around unit. Next, we assume that the term $(J-1) S_{t^{-}}$, which give the change before and after the jumps, is driven by increments $\mathrm{d} q_{t}$ of a Poisson process. Hence, from equation (11), setting $\alpha(t):=I(t)-G(t), \gamma(t):=\beta(t) / \alpha(t)$, we finally obtain the SDE (8).

\section{Mean-reversion condition}

A mean-reverting process has a drift term that brings the variable being pulled back to some equilibrium. This feature is captured by one stochastic differential equation if the following definition is verified.

Definition 1 (Condition $\left(A_{3}\right)$ of [22]) Consider a jumpdiffusion process $Y_{t}$ with a differentiable drift function $\mu($.). 
$\limsup _{\left|Y_{t}\right| \rightarrow \infty} \frac{\left|Y_{t}+\mu\left(Y_{t}\right)\right|}{\left|Y_{t}\right|}<1$,

then $Y_{t}$ is mean-reverting.

From this definition, we have the following proposition

Proposition 1 The jump-diffusion model (8) is mean-reverting.

Proof It is straightforward and is based on the fact that from an economic point of view, $\beta(t)$ is bounded on $[0, T]$ and we have $|1+\alpha(t)|<1$ for all $t \in[0, T]$.

\section{Regulated electricity forward price}

\section{Computation of regulated electricity forward price}

The price at time $t$ of the forward expiring at time $T$ (i.e., $\mathbf{F}(t, T))$ is obtained as the expected value of the spot price under an equivalent $\mathbb{Q}$-martingale measure, conditional on the information set available up to time $t$, precisely

$\mathbf{F}(t, T)=\mathbb{E}_{t}^{\mathbb{Q}}\left[S_{T}\right]$.

where $\mathbb{E}_{t}^{\mathbb{Q}}$ represents the conditional expectation knowing a natural filtration of $S_{t}$ under the risk-neutral probability $Q$. To incorporate the non-opportunity of arbitrage in the model, we use the same approach as in [20] and [3], which consists of incorporating a market price of risk in the drift, to obtain

$\widehat{\gamma}(t)=\gamma(t)-\lambda \frac{\sigma(t)}{\alpha(t)} S_{t}$,

where $\lambda$ denotes the market price of risk per unit risk linked to the state variable $S_{t}$. This market price of risk to be calibrated from market information pins down the choice of one particular martingale measure. Recall that when a market subjected to that measure, the opportunity of arbitrage is theoretically excluded in this market. Hence, under this equivalent martingale measure SDE (8) becomes

$\mathrm{d} S_{t}=-\alpha(t)\left(\widehat{\gamma}(t)-S_{t}\right) \mathrm{d} t+\sigma(t) S_{t} \mathrm{~d} \widehat{W}_{t}+(J-1) S_{t} \mathrm{~d} q_{t} ;$

substituting (12) in (13), we obtain

$$
\begin{aligned}
\mathrm{d} S_{t}= & -\alpha(t)\left(\gamma(t)-\left(1+\lambda \frac{\sigma(t)}{\alpha(t)}\right) S_{t}\right) \mathrm{d} t+\sigma(t) S_{t} \mathrm{~d} \widehat{W}_{t} \\
& +(J-1) S_{t} \mathrm{~d} q_{t},
\end{aligned}
$$

where $\mathrm{d} \widehat{W}$ is the increment of a Brownian motion in the $\mathbb{Q}$ -martingale measure specified by the choice of $\lambda$.
The next addresses the forward price computations.

Proposition 2 Assume that $J$, the increments of $q_{t}$ and $W_{t}$, are independent. Under the risk-neutral or martingale measure $\mathbb{Q}$ and Novikov hypothesis, i.e., $\mathbb{E}\left[\mathrm{e}^{\frac{1}{2} \int_{0}^{t} \sigma(s)^{2} \mathrm{~d} s}\right]<\infty$, electricity forward price under regulated market is given by

$\mathbf{F}(t, T)=S_{t} \mathrm{e}_{t}^{\int_{t}^{T}(\alpha(s)+\lambda \sigma(s)) \mathrm{d} s}-\int_{t}^{T} \beta(s) \mathrm{e}^{\int_{s}^{T}(\alpha(u)+\lambda \sigma(u)) \mathrm{d} u} \mathrm{~d} s$.

Before proving Proposition 2, let us first prove the following lemmas.

Lemma 1 The solution of equation (14) is the process $\left(S_{t}, 0 \leq t \leq T\right)$ defined by

$S_{t}=Z_{t}\left(S_{0}-\int_{0}^{t} \beta(s) Z_{s}^{-1} \mathrm{~d} s\right)$,

where $Z_{t}=\mathrm{e}^{\left(\int_{0}^{t}\left(\lambda \sigma(s)+\alpha(s)-\frac{1}{2} \sigma(s)^{2}\right) \mathrm{d} s+\int_{0}^{t} \sigma(s) \mathrm{d} \hat{W}_{s}+\int_{0}^{t} \ln J \mathrm{~d} q_{s}\right)}$.

Proof To solve equation (14), we consider a process $Z$, solution of the following equation

$\mathrm{d} Z_{t}=Z_{t}\left(\alpha(t)\left(1+\lambda \frac{\sigma(t)}{\alpha(t)}\right) \mathrm{d} t+\sigma(t) \mathrm{d} \widehat{W}_{t}+(J-1) \mathrm{d} q_{t}\right)$

and $Z_{0}=1$.

Applying Itô formula with jumps stated in 7.10 [9], we obtain

$Z_{t}=Z_{0} \mathrm{e}^{\left(\int_{0}^{t}\left(\lambda \sigma(s)+\alpha(s)-\frac{1}{2} \sigma(s)^{2}\right) \mathrm{d} s+\int_{0}^{t} \sigma(s) \mathrm{d} \hat{w}_{s}+\int_{0}^{t} \ln J \mathrm{~d} q_{s}\right)}$.

Now, let us set $f\left(S_{t}, Z_{t}\right)=\frac{S_{t}}{Z_{t}}$. By applying Itô formula with jumps one more, we obtain

$$
\begin{aligned}
\frac{S_{t}}{Z_{t}}= & \frac{S_{0}}{Z_{0}}+\int_{0}^{t} \frac{1}{Z_{s}}\left[\left((\lambda(s) \sigma(s)+\alpha(s)) \mathrm{d} s+\sigma(s) \mathrm{d} \widehat{W}_{s}\right.\right. \\
& \left.\left.+(J-1) \mathrm{d} q_{s}\right) S_{s}-\beta(s) \mathrm{d} s\right] \\
& -\int_{0}^{t} \frac{S_{s}}{\left(Z_{s}\right)^{2}} Z_{s}((\lambda(s) \sigma(s)+\alpha(s)) \mathrm{d} s \\
& \left.+\sigma(s) \mathrm{d} \hat{W}_{s}+(J-1) \mathrm{d} q_{s}\right) \\
& +\frac{1}{2}\left(\int_{0}^{t} 2 \frac{S_{s}}{\left(Z_{s}\right)^{3}}\left(\sigma(s) Z_{s}\right)^{2} \mathrm{~d} s-\frac{2}{\left(Z_{s}\right)^{2}} \sigma(s)^{2} S_{s} Z_{s} \mathrm{~d} s\right) .
\end{aligned}
$$

The development of (16) leads to 


$$
\begin{aligned}
\frac{S_{t}}{Z_{t}}= & \frac{S_{0}}{Z_{0}}+\int_{0}^{t} \frac{S_{s}}{Z_{s}}\left((\lambda(s) \sigma(s)+\alpha(s)) \mathrm{d} s+\sigma(s) \mathrm{d} \widehat{W}_{s}\right. \\
& \left.+(J-1) \mathrm{d} q_{s}\right)-\int_{0}^{t} \frac{1}{Z_{s}} \beta(s) \mathrm{d} s \\
& -\int_{0}^{t} \frac{S_{s}}{Z_{s}}((\lambda(s) \sigma(s)+\alpha(s)) \mathrm{d} s \\
& \left.+\sigma(s) \mathrm{d} \widehat{W}_{s}+(J-1) \mathrm{d} q_{s}\right) \\
& +\int_{0}^{t} \frac{S_{s}}{Z_{s}} \sigma(s)^{2} \mathrm{~d} s-\frac{S_{s}}{Z_{s}} \sigma(s)^{2} \mathrm{~d} s .
\end{aligned}
$$

By observing that the sum of the first and fourth term of equation (17) is equal to zero and since $Z_{0}=1$ we obtain

$\frac{S_{t}}{Z_{t}}=S_{0}-\int_{0}^{t} Z_{s}^{-1} \beta(s) \mathrm{d} s$.

Finally, we obtain

$S_{t}=Z_{t} S_{0}-Z_{t} \int_{0}^{t} Z_{s}^{-1} \beta(s) \mathrm{d} s$.

This ends the proof. by

Furthermore, the solution of (14) at $T$ starting at $t$ is given

$S_{T}=\frac{Z_{T}}{Z_{t}} S_{t}-Z_{T} \int_{t}^{T} \alpha(s) \mu(s) Z_{s}^{-1} \mathrm{~d} s$,

where $\left.Z_{T}=Z_{t} \mathrm{e}^{\left(\int_{t}^{T}\left(\lambda \sigma(s)+\alpha(s)-\frac{1}{2} \sigma(s)^{2}\right)\right.} \mathrm{d} s+\int_{t}^{T} \sigma(s) \mathrm{d} \widehat{w}_{s}+\int_{t}^{T} \ln J \mathrm{~d} q_{s}\right)$.

Lemma 2 If $J$ is a log-normal distributed process with $\mathbb{E}[J]=1$ and $q$ a Poisson process, then $\mathbb{E}_{t}^{\mathbb{Q}}\left[\mathrm{e}^{\int_{t}^{T} \ln J_{s}} \mathrm{~d}_{s}\right]=1$.

Proof Firstly, we use differentiation method to compute $\mathbb{E}_{t}^{\mathbb{Q}}\left[\mathrm{e}^{\int_{0}^{t} \ln J_{s} \mathrm{~d} q_{s}}\right]$.

Let us define $L_{t}$ such that

$$
\begin{aligned}
L_{t} & \equiv \mathrm{e}^{\int_{0}^{t} \ln J_{s} \mathrm{~d} q_{s}}, \\
& \equiv \mathrm{e}^{m_{t}}
\end{aligned}
$$

where $m_{t}$ is

$m_{t}=\int_{0}^{t} \ln J_{s} \mathrm{~d} q_{s}$,

or equivalently $\mathrm{d} m_{t}=\ln J_{t} \mathrm{~d} q_{t}$.

In order to write the dynamic followed by $L_{t}$ for process define in (20) we use the generalization form of Itô's lemma [9]. The SDE verified by $L_{t}$ is

$$
\begin{aligned}
L_{t}= & L_{0}+\int_{0}^{t} L_{s} \ln J_{s} \mathrm{~d} q_{s} \\
& -\int_{0}^{t} L_{s} \ln J_{s} \mathrm{~d} q_{s}+\int_{0}^{t} L_{s}\left(\mathrm{e}^{\ln J_{s}}-1\right) \mathrm{d} q_{s} \\
= & 1+\int_{0}^{t} L_{s}\left(\mathrm{e}^{\ln J_{s}}-1\right) \mathrm{d} q_{s} .
\end{aligned}
$$

Then, from (21) we obtain

$$
\begin{aligned}
\mathbb{E}\left[L_{t}\right] & =1+\int_{0}^{t} \mathbb{E}\left[L_{s}\right]\left(\mathbb{E}\left[\mathrm{e}^{\ln J_{s}}\right]-1\right) \ell \mathrm{d} s \\
& =1 .
\end{aligned}
$$

Alternatively, we can remark that $\int_{0}^{t} \ln J_{s} \mathrm{~d} q_{s}=\sum_{i=0}^{q_{t}} \ln J_{s}$ which is the particular case of Lévy process with the moment-generating function. Using Lévy-Khintchine representation, we have

$$
\begin{aligned}
\mathbb{E}\left[\mathrm{e}^{i u \int_{0}^{t} \ln J_{s} \mathrm{~d} q_{s}}\right] & =\mathbb{E}\left[\mathbb{E}\left[\mathrm{e}^{i u \int_{0}^{t} \ln J \mathrm{~d} q_{s}} \mid q_{t}\right]\right] \\
& =\mathbb{E}\left[\mathbb{E}\left[\varphi(u)^{q_{t}}\right]\right] \\
& =\mathrm{e}^{t \ell(\varphi(u)-1)} .
\end{aligned}
$$

where $\varphi$ is the moment-generating function of the jump $\ln J$. Evaluating (22) at $u=-i$ leads to desired result.

Proof of Proposition 2 Before stating let us recall that forward price formula is given by $\mathbf{F}(t, T)=\mathbb{E}_{t}^{\mathbb{Q}}\left[S_{T}\right]$. By substituting $S_{T}$ with (18), we obtain

$$
\begin{aligned}
& \mathbf{F}(t, T)=\mathbb{E}_{t}^{\mathbb{Q}}\left[S_{T}\right] \\
& =S_{t} \mathbb{E}_{t}^{\mathbb{Q}}\left[\mathrm{e}^{\left(\int_{t}^{T}\left(\lambda \sigma(s)+\alpha(s)-\frac{1}{2} \sigma(s)^{2}\right) \mathrm{d} s+\int_{t}^{T} \sigma(s) \mathrm{d} \hat{W}_{s}+\int_{t}^{T} \ln J_{s} \mathrm{~d} q_{s}\right)}\right]
\end{aligned}
$$

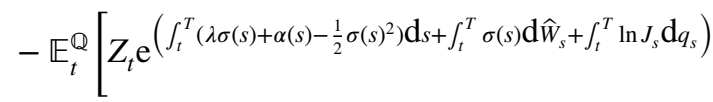

$$
\begin{aligned}
& \left.\times \int_{t}^{T} \alpha(s) \mu(s) Z_{s}^{-1} \mathrm{~d} s\right] .
\end{aligned}
$$

We first compute $\mathbb{E}_{t}^{\mathrm{Q}}\left[\mathrm{e}^{\left(\int_{t}^{T}\left(\lambda \sigma(s)+\alpha(s)-\frac{1}{2} \sigma(s)^{2}\right) \mathrm{d} s+\int_{t}^{T} \sigma(s) \mathrm{d} \hat{W}_{s}+\int_{t}^{T} \ln J_{s} \mathrm{~d} \mathrm{~d}_{s}\right)}\right] \equiv A$. 
Fig. 1 Spot prices for the parameters, $I=0.0314$; $G=0.01 ; E=0.05 ; H=0.001 ;$ $F=0.05 ; \ell=2.85 ; \sigma=0.75$; $\sigma J=0.67 ; S(0)=50$

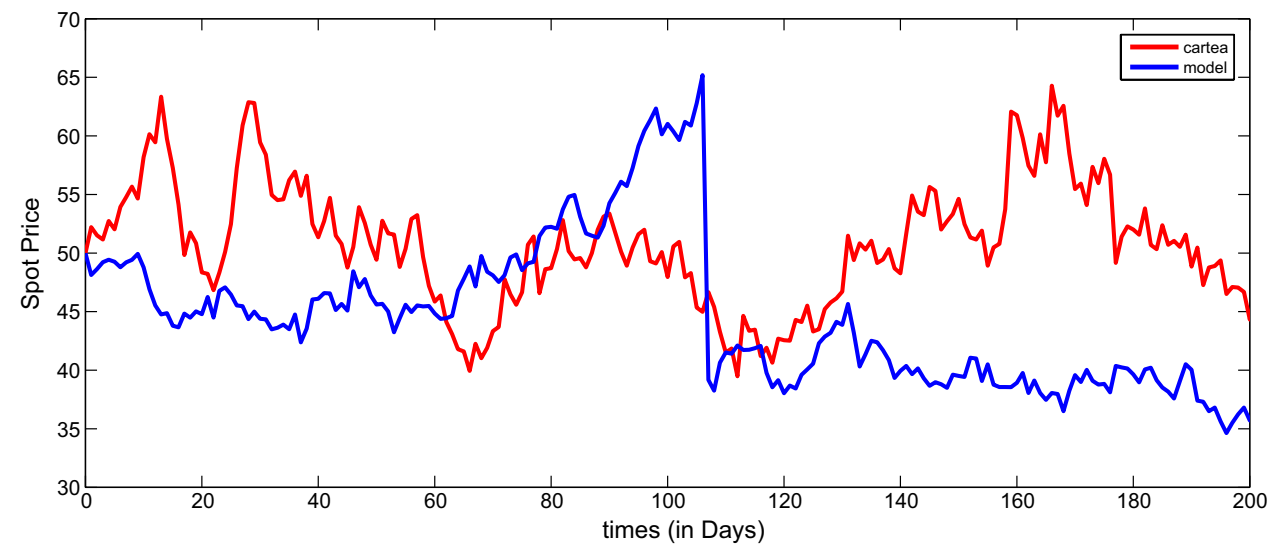

From independence between $J, \mathrm{~d} q_{t}$ and $\mathrm{d} W_{t}$, we obtain

$$
\begin{aligned}
A & =\mathbb{E}_{t}^{\mathbb{Q}}\left[\mathrm{e}^{\left(\int_{t}^{T}\left(\lambda \sigma(s)+\alpha(s)-\frac{1}{2} \sigma(s)^{2}\right) \mathrm{d} s+\int_{t}^{T} \sigma(s) \mathrm{d} \hat{W}_{s}\right)}\right] \mathbb{E}_{t}^{\mathbb{Q}}\left[\mathrm{e}^{\int_{t}^{T} \ln J \mathrm{~d} q_{s}}\right] \\
& =\mathrm{e}^{\int_{t}^{T}(\alpha(s)+\lambda \sigma(s)) \mathrm{d} s} \mathbb{E}_{t}^{\mathbb{Q}}\left[\mathrm{e}^{\left.\int_{t}^{T} \ln J \mathrm{~d} q_{s}\right]}\right.
\end{aligned}
$$

We now compute

$$
\mathbb{E}_{t}^{\mathbb{Q}}\left[Z_{t} \mathrm{e}^{\left(\int_{t}^{T}\left(\lambda \sigma(s)+\alpha(s)-\frac{1}{2} \sigma(s)^{2}\right) \mathrm{d} s+\int_{t}^{T} \sigma(s) \mathrm{d} \hat{w}_{s}+\int_{t}^{T} \ln J \mathrm{~d} q_{s}\right)} \int_{t}^{T} \alpha(s) \gamma(s) Z_{s}^{-1} \mathrm{~d} s\right] \equiv A_{1} .
$$

Replacing $Z_{s}^{-1}$ by its expression, using independence between $J, \mathrm{~d} q_{t}$ and $\mathrm{d} W_{t}$ and Fubini theorem [29], we obtain

$$
\begin{aligned}
F(t, T)= & G(T)\left(\frac{S(t)}{G(t)}\right)^{\mathrm{e}^{-\alpha(T-t)}} \\
& \mathrm{e}^{\int_{t}^{T} \frac{1}{2} \sigma^{2}(s) \mathrm{e}^{-2 \alpha(T-s)}-\lambda \sigma(s) \mathrm{e}^{-\alpha(T-s)} \mathrm{d} s+\int_{t}^{T} \xi\left(\sigma_{J}, \alpha, T, s\right) \ell \mathrm{d} s-\ell(T-t)}
\end{aligned}
$$

The forward price formula (15) derived in this work is an affine function of the spot price $S_{t}$, Unlike in the works of [3], where they have obtained a power function of the spot price. This is what justifies the presence of fewer jumps in the forward prices. This is in line with the fact that we are in a regulating context where prices are likely to undergo less variations.

$$
\begin{aligned}
& A_{1}=\mathbb{E}_{t}^{\mathbb{Q}}\left[\int_{t}^{T} \alpha(s) \gamma(s) \mathrm{e}^{\left(\int_{s}^{T}\left(\lambda \sigma(u)+\alpha(u)-\frac{1}{2} \sigma(u)^{2}\right) \mathrm{d} u+\int_{s}^{T} \ln J \mathrm{~d} q_{u}+\int_{s}^{T} \sigma(u) \mathrm{d} \widehat{W}_{u}\right)} \mathrm{d} s\right] \\
& =\int_{t}^{T} \mathbb{E}_{t}^{\mathbb{Q}}\left[\mathrm{e}^{\int_{s}^{T} \ln J \mathrm{~d} q_{u}}\right] \alpha(s) \gamma(s) \mathbb{E}_{t}^{\mathbb{Q}}\left[\mathrm{e}^{\left(\int_{s}^{T}\left(\lambda \sigma(u)+\alpha(u)-\frac{1}{2} \sigma(u)^{2}\right) \mathrm{d} u+\int_{s}^{T} \sigma(u) \mathrm{d} \hat{W}_{u}\right)}\right] \mathrm{d} s \\
& =\int_{t}^{T} \alpha(s) \gamma(s) \mathrm{e}^{\int_{s}^{T}(\alpha(u)+\lambda \sigma(u)) \mathrm{d} u} \mathrm{~d} s .
\end{aligned}
$$

By replacing finally (24) and (25) in (23), we obtain the forward price

$\mathbf{F}(t, T)=S_{t} \mathrm{e}^{\int_{t}^{T}(\alpha(s)+\lambda \sigma(s)) \mathrm{d} s}-\int_{t}^{T} \beta(s) \mathrm{e}^{\int_{s}^{T}(\alpha(u)+\lambda \sigma(u)) \mathrm{d} u} \mathrm{~d} s$.

This completes the proof.

\section{Analytical comparison with forward price in Cartea et al. (2005)}

Recall that forward price obtained in [3] is given by

\section{Some illustrative curves of spot and forward price in regulated electricity market}

This section deals with the numerical simulations of the forward price in order to illustrate some meaningful behaviors of the model and in comparison with the model developed in [3]. The proposed simulations also aim at highlighting the fundamental role of some particular parameters in the outcomes of the prices. For the numerical computation of spot and forward prices, we approximated the integrals using the trapezium and the Stratonovich integration methods. The parameters used in the simulations are plausible relative to those used in the literature. 
Fig. 2 Spot prices for the parameters, $I=0.0314$; $G=0.01 ; E=0.05 ; H=0.001 ;$ $F=0.05 ; \ell=8.85 ; \sigma=0.75$; $\sigma J=0.67 ; S(0)=50$

Fig. 3 Spot prices for the parameters, $I=0.0314 ; G=0.01$;

$E=0.05 ; H=0.001 ; F=0.05$

$\ell=1.5 ; \sigma=\exp (-0.015 t)$

$\sigma J=0.67 ; S(0)=50$
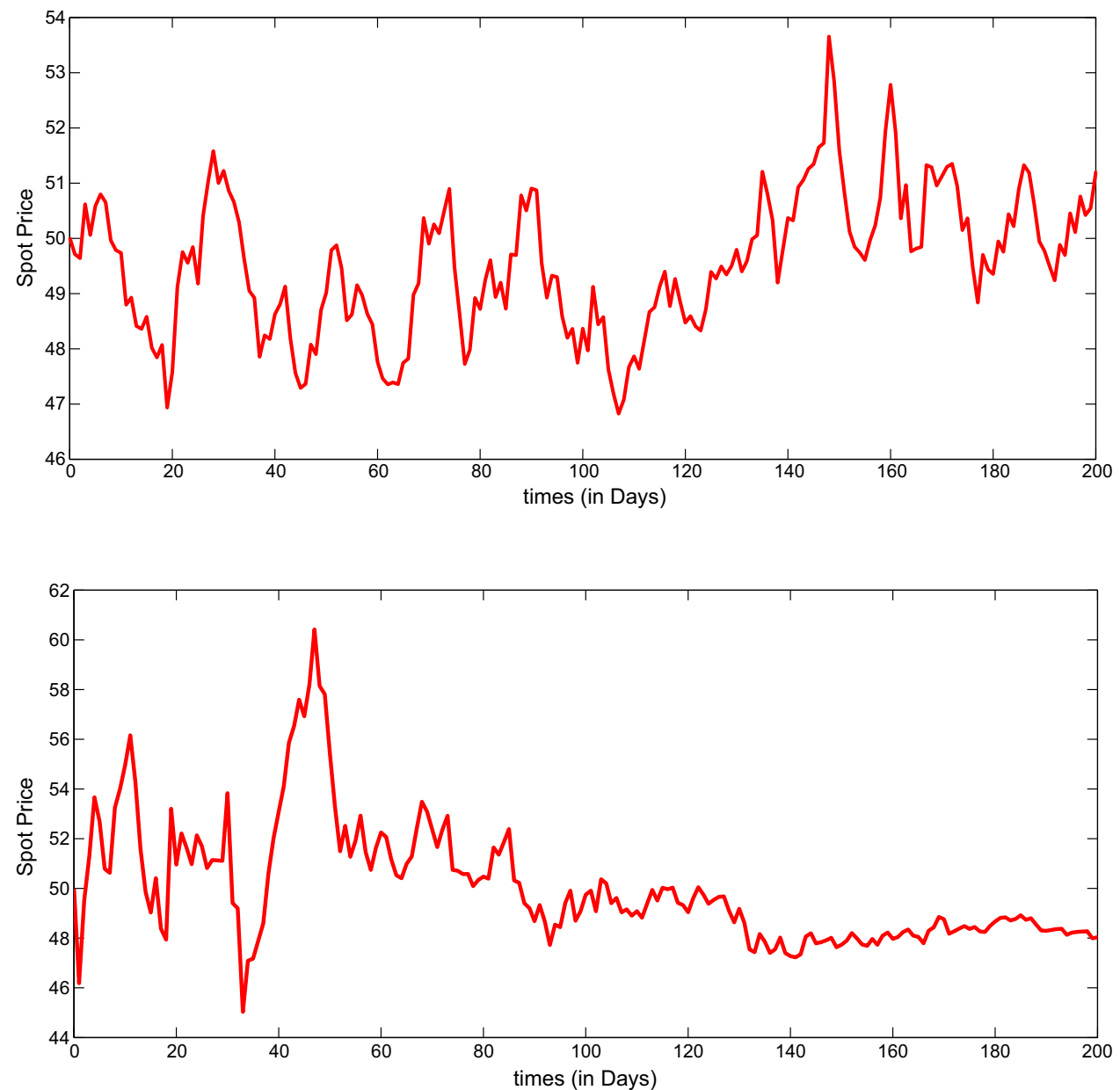

Figure 1 shows a simulated spot price compared to the spot price in [3] without the seasonal part. One can observe that the proposed model captures some characteristics discussed in the regulated market such as mean reversion, a property also observed in Figs. 2 and 3 confirming the theoretical results. It is further relevant to discuss that in our model, the frequency of jumps is less than in the deregulated market. Figures 4 and 5 present four different states of the evolution of the forward price process in the absence of jumps in the spot price model. Here, we observe that the forward price fluctuates around an average like the spot price. This could be justified by the fact that the forward formula obtained here is a functional of the spot price. Figure 6 obtained by introducing small jumps into the model shows that despite the jump at the beginning, the forward price latter oscillates around an equilibrium a situation which is not observed in Fig. 7 with bigger jumps. In a nut shell, these illustrations show that our model with the mean-reversion property captures the main objective of regulation principle, which is to cap prices within a given range.
Figure 8 shows that despite jumps in the prices, prices vary from a certain threshold for different maturities. We observe in Fig. 9 that when the efficiency rate factor $G$ is more than the inflation rate factor $I$, forward price decreases over time. This is in accordance with the economic principle.

\section{Conclusion}

In this paper, we have proposed a new model of spot price in regulated electricity market. The principal aim was to propose the forward price in regulated electricity market using economic principle price cap. It is also motivated by the fact that incentives regulation in public utilities, especially in electricity field, becomes more prevalent. The proposed model leads to non-classical Ornstein-Uhlenbeck process due to non-constant speed of the mean reversion. The determination of the exact solution permits us to derive the explicit expression of the forward price. An important topic for further research is to use historical data to calibrate the introduced model. 
(a)

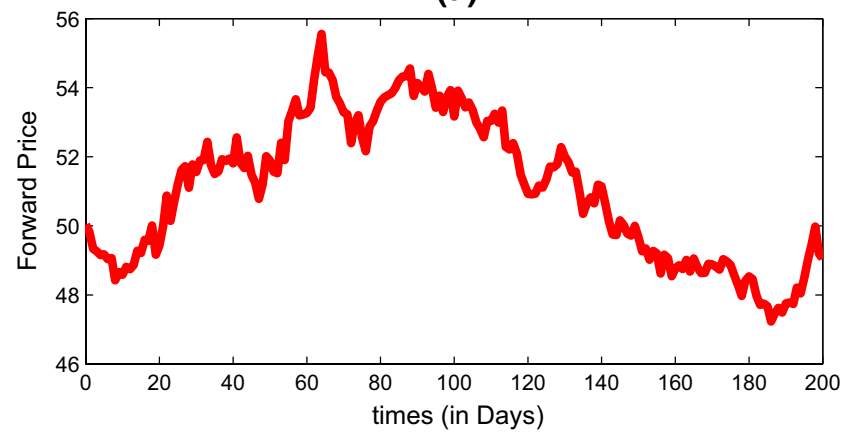

(c)

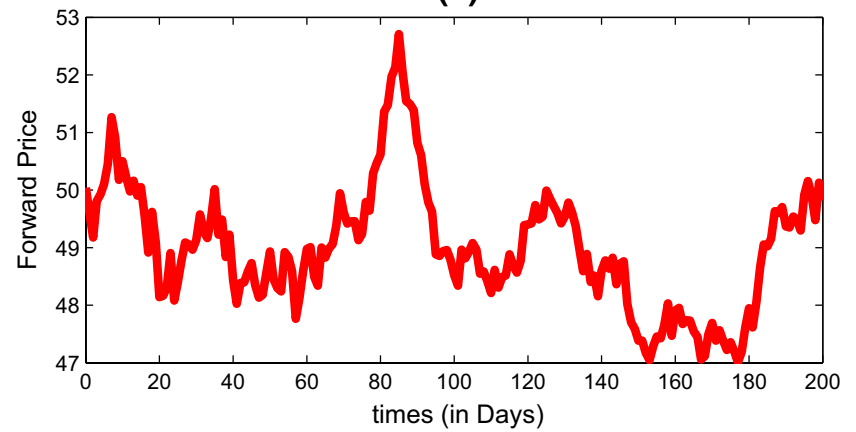

(b)

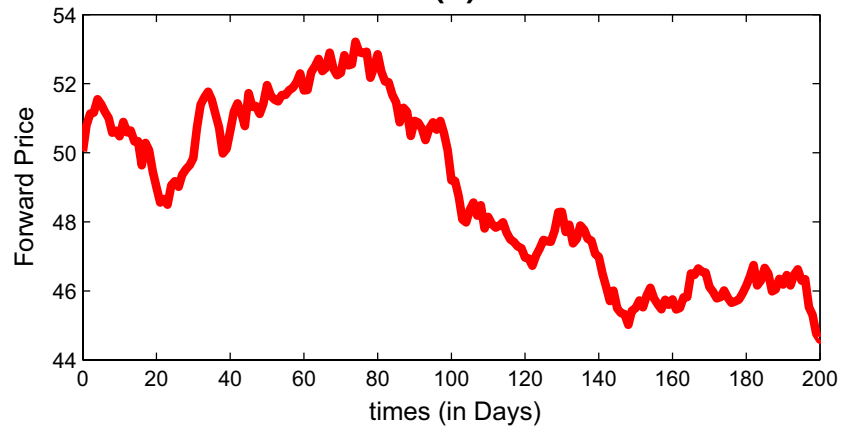

(d)

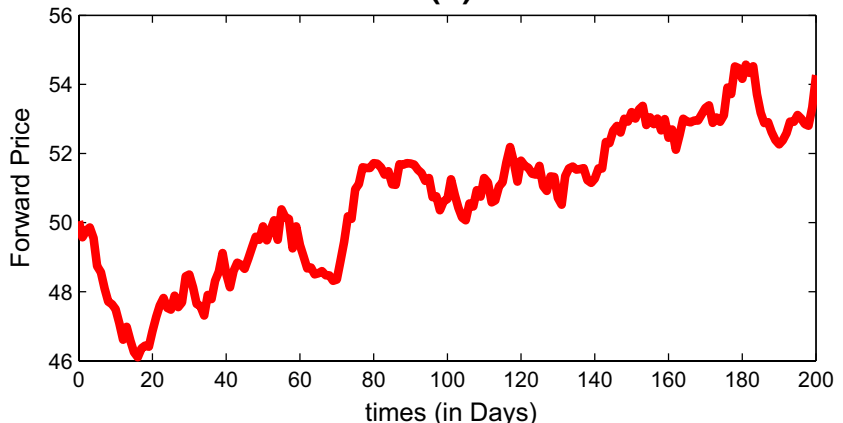

Fig. 4 Forward price for the parameters, $I=0.0314 ; G=0.01 ; E=0.05 ; H=0.001 ; F=0.05 ; \sigma=0.75 ; \sigma J=0.67 ; S(0)=50$

(a)

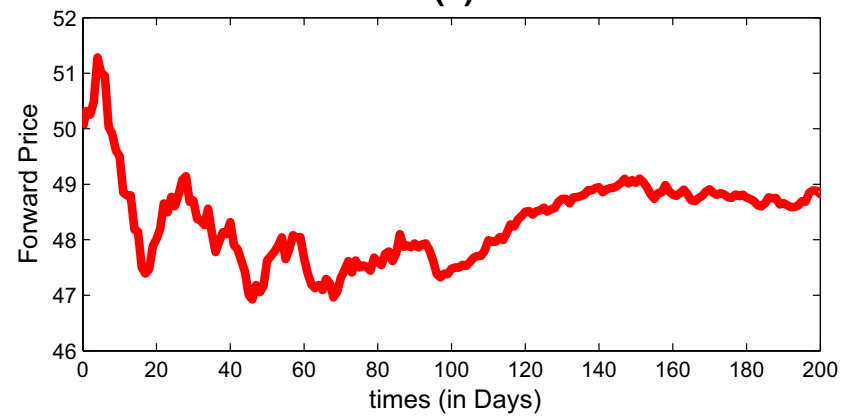

(c)

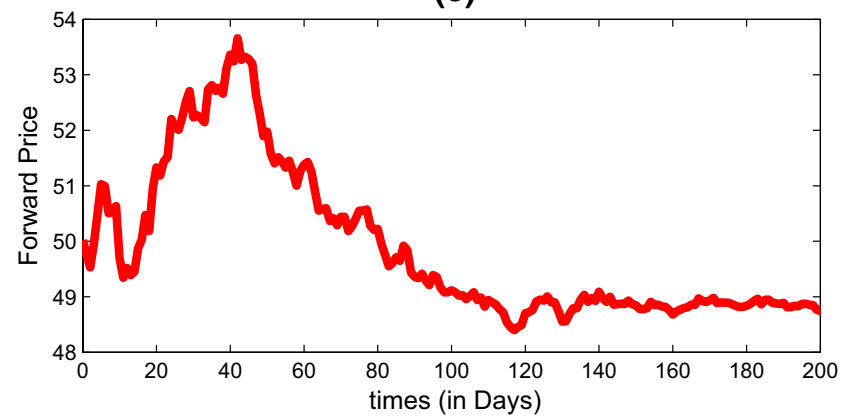

(b)

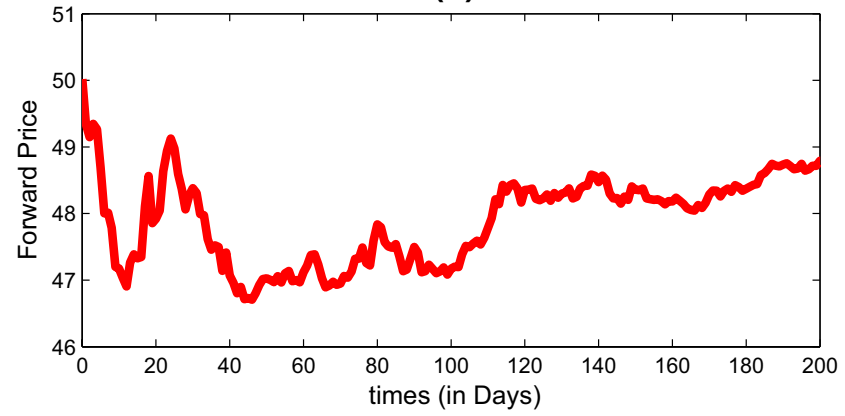

(d)

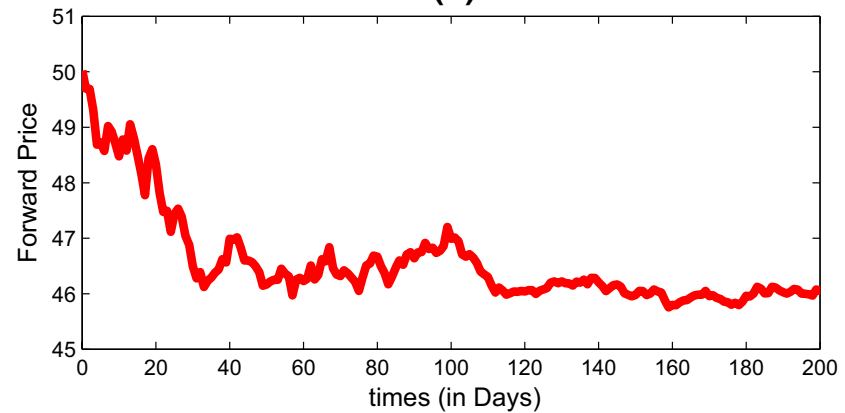

Fig. 5 Forward prices for the parameters, $I=0.0314 ; G=0.01 ; E=0.05 ; H=0.001 ; F=0.05 ; \sigma=\exp (-0.01 t) ; \sigma J=0.67 ; S(0)=50$ 
(a)

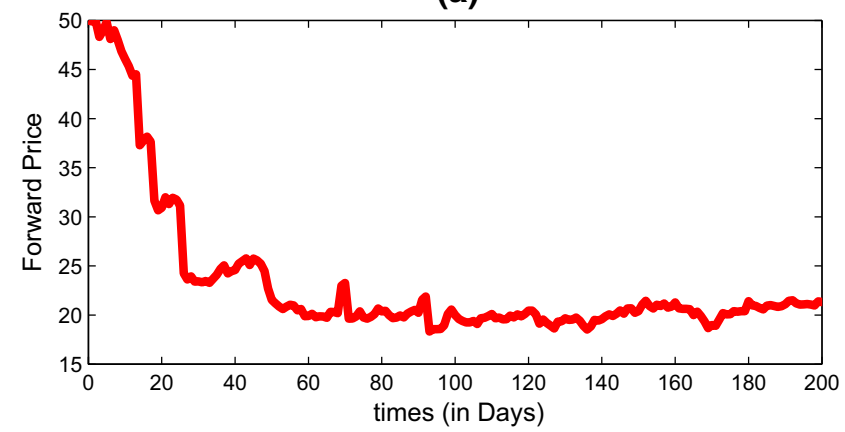

(c)

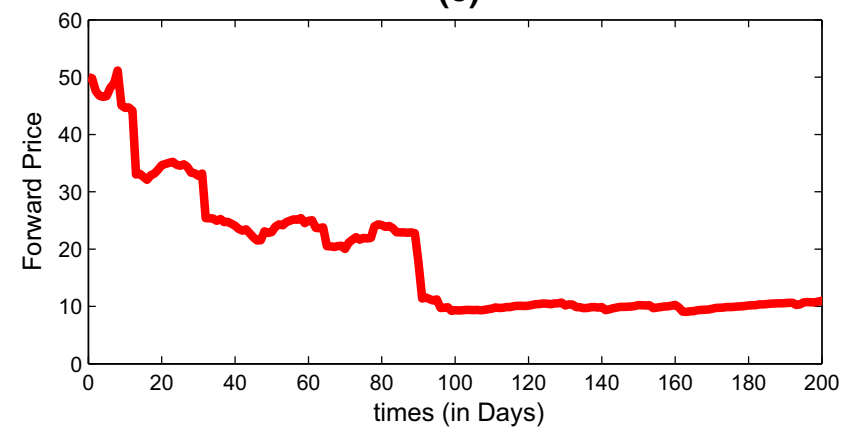

(b)

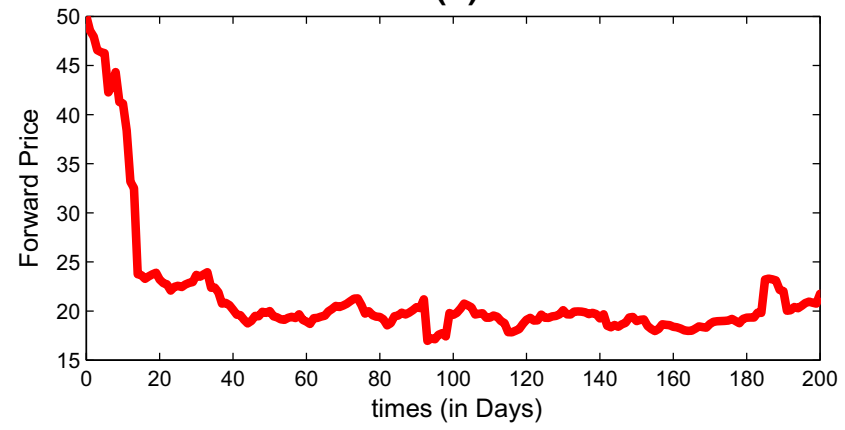

(d)

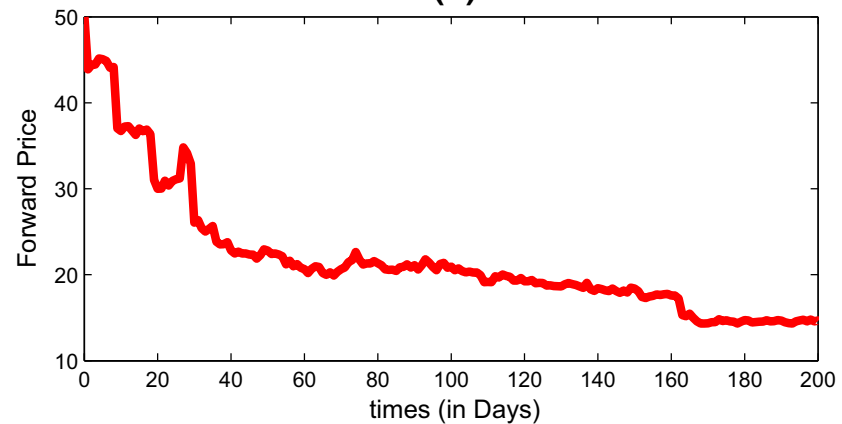

Fig. 6 Forward prices for the parameters, $I=0.0314 ; G=0.01 ; E=0.05 ; H=0.001 ; F=0.05 ; \ell=8.5 ; \sigma=0.75 ; \sigma J=0.67 ; S(0)=50$

(a)

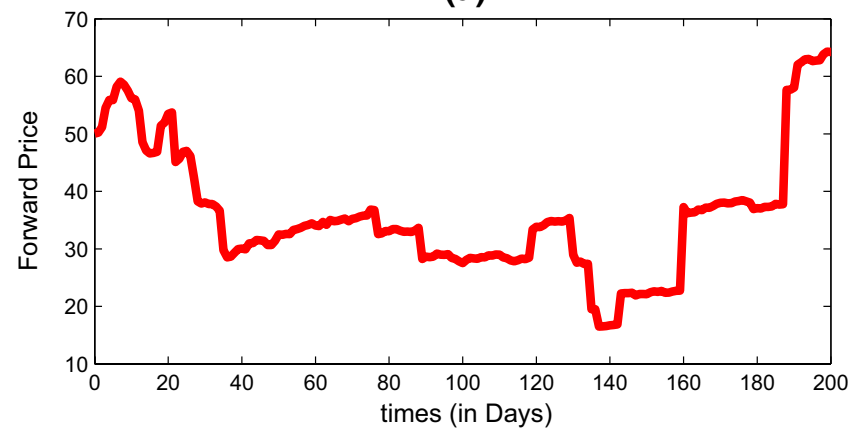

(c)

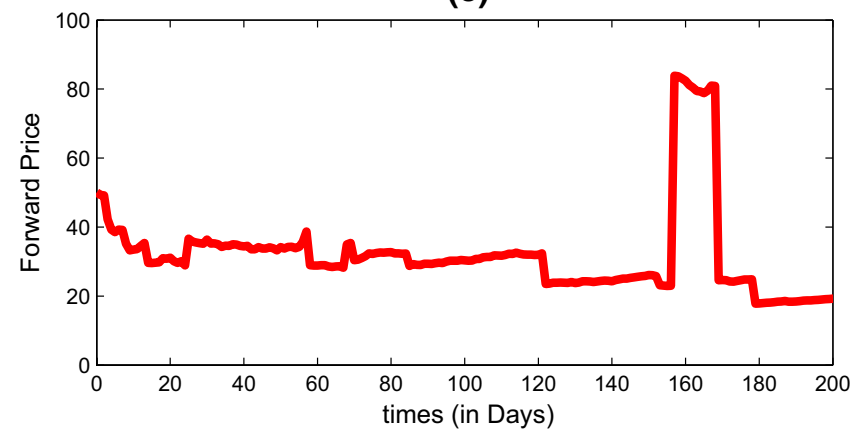

(b)

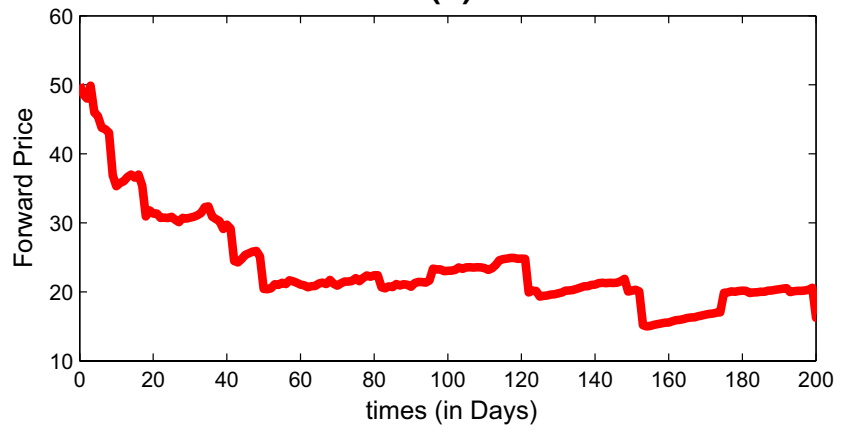

(d)

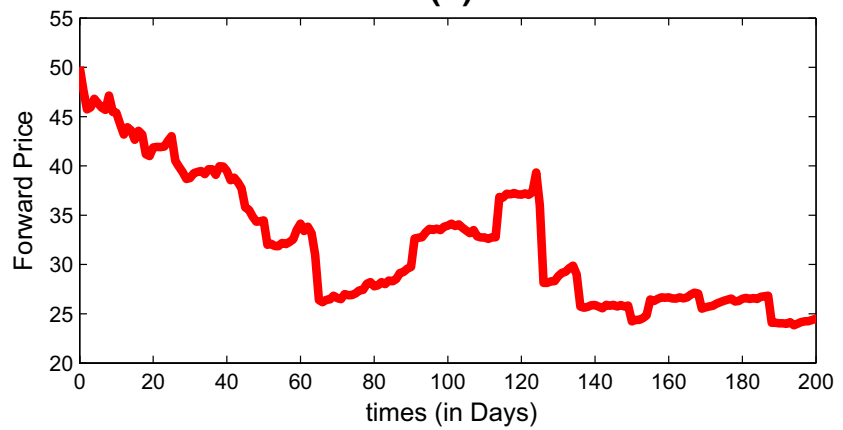

Fig. 7 Forward prices for the parameters, $I=0.0314 ; G=0.01 ; E=0.05 ; H=0.001 ; F=0.05 ; \ell=8.5 ; \sigma=\exp (-0.01 t) ; \sigma J=0.67 ; S(0)=50$ 


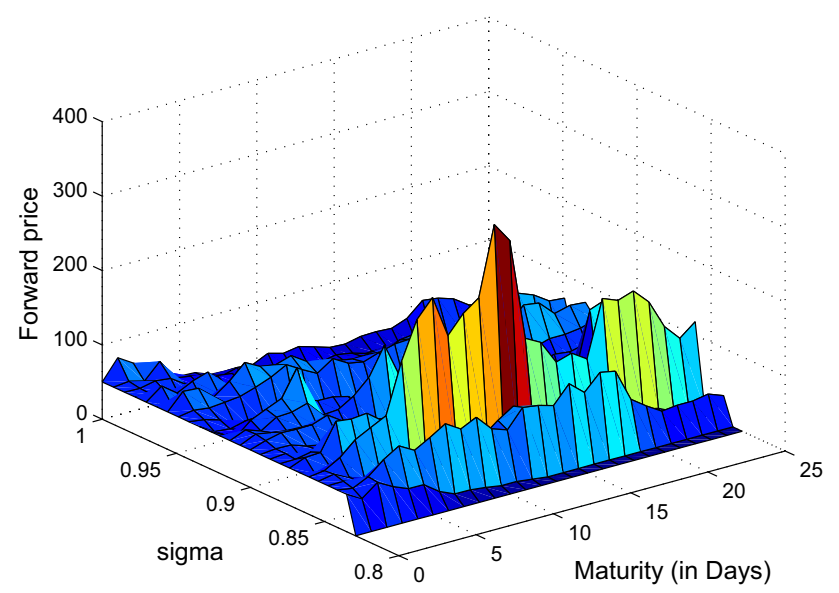

Fig. 8 Forward prices for each day for various maturities and parameter sigma, $\quad I=0.0314 ; G=0.01 ; \quad E=0.05 ; H=0.001$; $F=0.05 ; \ell=8.5 ; \sigma=\exp (-0.01 t) ; \sigma J=0.67 ; S(0)=50$

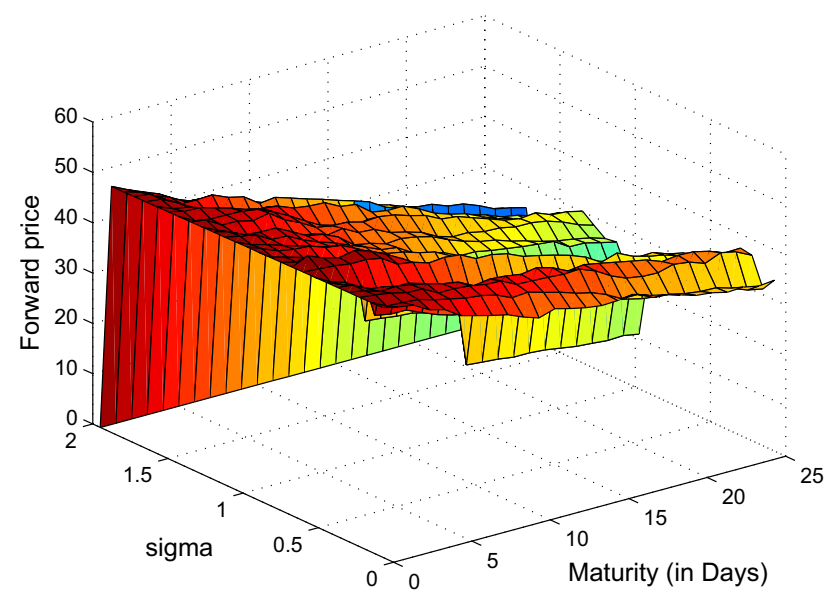

Fig. 9 Forward prices for each day for various maturities and parameter sigma $I=0.0314 ; G=0.1 ; S(0)=50 ; \sigma J=0.67 ; \ell=0.25$

Acknowledgements We thank the African Center of Excellence in Technologies, Information and Communication (CETIC) to place in our disposal its infrastructures. This helps us to improve conditions of this work. We would like to thank anonymous referees for their helpful comments which ultimately improved the article.

Open Access This article is distributed under the terms of the Creative Commons Attribution 4.0 International License (http://creativeco mmons.org/licenses/by/4.0/), which permits unrestricted use, distribution, and reproduction in any medium, provided you give appropriate credit to the original author(s) and the source, provide a link to the Creative Commons license, and indicate if changes were made.

\section{References}

1. Acton, J., Vogelsang, I.: Symposium on price-cap regulation: introduction. Rand J. Econ. 20, 369 (1989)
2. Aid, R., Campi, L., Langrené, N.: A structural risk-neutral model for pricing power derivatives, hal-00525800, version 1-12 (2010)

3. Cartea, A., Figueroa, G.M.: Pricing in electricity markets: a mean reverting jump diffusion model with seasonality. Appl. Math. Finance 12(4), 313-335 (2005)

4. Chanez, C., Signarbieux, B., Coutan, F.: Tarification de l'électricité:hausse des prix progressive. Atlante \& Cie, Neuillysur-Seine (2012)

5. Clewlow, L., Strickland, C.: Energy Derivatives, Pricing and Risk Management. Lacima Publications, London (2000)

6. Clewlow, L., Strickland, C., Kaminski, V.: Extending MeanReversion Jump Diffusion. Energy Power Risk Management. Risk Waters Group, London (2001)

7. Deng, S.: Stochastic Models of Energy Commodity Prices and Their Applications: Mean-reversion with Jumps and Spikes. Working paper, Georgia Institute of Technology (2000)

8. Schwartz, E.: The stochastic behavior of commodity prices: implications for valuation and hedging. J. Finance 52(3), 923 973 (1997)

9. Etheridge, A.: A Course in Financial Calculus, 1st edn. Cambridge University Press, Cambridge (2002)

10. Escribano A., Pena J.I., Villaplana P.: Modelling electricity prices: inter-national evidence. Working paper (2002)

11. ENMAX, Power Corporation 2007-2016 formula Based Ratemaking, Alberto Utilities Commission, Decision N0. 2009035 (Mar. 25, 2009)

12. Roques, F.A., Savva, N.S.: Price Cap Regulation and Investment Incentive Demand Uncertainty. Working paper (2006)

13. Hanson, F.B.: Applied Stochastic Processes and Control for JumpDiffusions: Modeling, Analysis and Computation. Society for Industrial and Applied Mathematics, Philadelphia (2007)

14. Geman, H., Roncoroni, A.: Understanding the structure of electricity prices. J. Bus. 1261, 79-1225 (2003)

15. Geman, H., Roncoroni, A.: Understanding the fine structure of electricity prices. J. Bus. 79(3), 1225-1262 (2006)

16. Joskow, P.L.: Incentive regulation and its application to electricity networks. Rev. Netw. Econ. 7(4), 1-14 (2008)

17. Joskow, P.L.: Incentive Regulation in Theory and Practice: Electricity Distribution And Transmission Network, Prepared for the National bureau of Economic Research on Economic Conference Regulation, Working Paper 05-18, Brooking Joint Center for Regulatory Studies, Washington, DC (2005)

18. Laffont, J.-J., Tirole, J.: A Theory of Incentives in Procurement and Regulation. MIT Press, Cambridge (1993)

19. Littlechild, S.: Regulation of British Telecommunication' Profitability. Tech. Rep., Department of Industry, London (1983)

20. Lucia, J.J., Schwartz, S.S.: Electricity prices and power derivatives: evidence from the Nordic Power Exchange. Rev. Deriv. Res. 5, 5-50 (2002)

21. McDermott, K.L.: Changing regulatory incentives in reinventing electric utility regulation. In: Enholm, G., Malko, J.R. (eds.) Public Utility Report, Inc., Viennia, VA, pp. 179-198 (1995)

22. Mbele Bidima, M.L.D., Rásonyi, M.: Asymptotic Exponential Arbitrage and Utility-Based Asymptotic Arbitrage in Markovian Models of Financial Markets. Springer, vol. 138, pp. 1-15 (2014)

23. Merton, R.M.: Continuous Times-Finance. First revised edition. Blackwell, Oxford (2001)

24. Meyer-Brandis, T., Tankov, P.: Multi-factor jump-diffusion models of electricity prices. Int. J. Theor. Appl. Finance 11, 503-528 (2008)

25. Nazarova, A.: Lévy-Based Electricity Spot Price Modelling, Master thesis, University of Ulm (2008)

26. Piebalgs, A.: De l'Energie pour le Développement Atteindre des Résultats dans la décennie de l'Energie Durable pour tous, europaid/energy (2014)

27. Phillips, C.F.: The Economic of Rgegulation. PUR, Vienna (1985) 
28. Polipovic, D.: Energy Risk: Valuing and Managing Energy Derivatives. Mac-Graw Hill, New York (2007)

29. Verrar, M.: The Stochastic Fubini Theorem Revisited. Delft Institute of Applied Mathematics, Delft University of Technology, Delft (2000)

30. Villaplana, P.: Pricing power derivatives: a two factor jump diffusion approach, Working Paper 03-18 Business Economics Series
05 Departamento de Economía de la Empresa Universidad Carlos III de Madrid (2003)

Publisher's Note Springer Nature remains neutral with regard to jurisdictional claims in published maps and institutional affiliations. 